\title{
FUNCTIONAL LIMIT THEOREMS IN HÖLDER SPACE FOR RESIDUALS OF NEARLY NONSTATIONARY AR(1) PROCESS
}

BY

\author{
JURGITA MARKEVIČIŪTĖ (VILNIUS)
}

\begin{abstract}
We investigate the polygonal line process built on the residuals of the first order nearly nonstationary autoregressive process. We prove functional limit theorems in Hölder space in two cases: the autoregressive coefficient $\phi_{n}$ is defined as $e^{\gamma / n}, \gamma<0$ is a constant, and $\phi_{n}$ is defined as $1-\gamma_{n} / n, \gamma_{n} \rightarrow \infty$, and $\gamma_{n} / n$ tends to zero as $n \rightarrow \infty$. Also we discuss some applications of these functional limit theorems in epidemic change detection.
\end{abstract}

2010 AMS Mathematics Subject Classification: Primary: 60F17; Secondary: 62M10.

Key words and phrases: Nearly nonstationary process, residuals, Hölderian functional limit theorems, epidemic change.

\section{INTRODUCTION}

We analyze the polygonal line process built on the least squares residuals of a first order nearly nonstationary autoregressive process $\left(y_{n, k}: k=1, \ldots, n\right.$; $n=1,2, \ldots)$ given by

$$
y_{n, k}=\phi_{n} y_{n, k-1}+\varepsilon_{k},
$$

where $0<\phi_{n}<1$ for fixed $n, \phi_{n} \rightarrow 1$ as $n \rightarrow \infty,\left(\varepsilon_{k}\right)$ is a sequence of independent identically distributed random variables with $\mathbb{E} \varepsilon_{k}=0, \mathbb{E} \varepsilon_{k}^{2}=1$ and $y_{n, 0}=0$.

The partial sums process of residuals was investigated by various authors because of its applicability in many statistical areas, like detecting structural changes or estimating probability density. A lot of studies are made for residuals partial sums of stationary and nonstationary autoregression model. For stationary processes, the weak limits of partial sums of autoregressive model's residuals were studied by Kulperger [1]], and ARMA model's residuals by Bai [2]. Horváth [8] and Bai [2] proposed applications of limit theorems to change-point problems. Other authors analyzed more general processes based on residuals. For example, $\mathrm{Yu}$ [25] and Kulperger and $\mathrm{Yu}$ [12] constructed high moment partial sum processes 
based on residuals of ARMA and GARCH models, respectively. The residuals of stationary and nonstationary $\mathrm{AR}(1)$ process were studied by Shin [23]. He found that residuals partial sums processes converge to a standard Brownian motion when the autoregressive coefficient is strictly less than one, and it is a randomly shifted Brownian motion when the coefficient is equal to one.

Some functionals of the paths of the residuals partial sums processes are used as test statistics for the null hypothesis under certain alternatives. So one is interested in a larger choice of possible functionals, because then one has a bigger class of possible alternatives. The use of Hölder space provides functional limit theorems of a wider scope (see, e.g., Juodis et al. [10]). Račkauskas and Rastenè [19] extended Shin's [23] results establishing the convergence in Hölder spaces of polygonal line processes constructed from partial sums of residuals of the $\operatorname{AR}(1)$ model.

We establish the convergence in Hölder spaces of the polygonal line processes $\widehat{W}_{n}=\left(\widehat{W}_{n}(t), t \in[0,1], n>0\right)$ built on the least squares residuals $\left(\widehat{\varepsilon}_{k}\right)$ :

$$
\begin{aligned}
& \widehat{W}_{n}(t):=\sum_{k=1}^{[n t]} \widehat{\varepsilon}_{k}+(n t-[n t]) \widehat{\varepsilon}_{[n t]+1}, \quad \widehat{W}_{n}(0)=0 \quad \text { and } \\
& \widehat{W}_{n}(t)=0 \quad \text { if } \sum_{k=1}^{n} y_{n, k-1}^{2}=0
\end{aligned}
$$

The residuals of the model (ㅁ. $)$ are defined by

$$
\widehat{\varepsilon}_{k}=y_{n, k}-\widehat{\phi}_{n} y_{n, k-1}=\varepsilon_{k}-\left(\widehat{\phi}_{n}-\phi_{n}\right) y_{n, k-1},
$$

where $\widehat{\phi}_{n}$ is the least squares estimate of the coefficient $\phi_{n}$ defined by

$$
\widehat{\phi}_{n}=\frac{\sum_{k=1}^{n} y_{n, k} y_{n, k-1}}{\sum_{k=1}^{n} y_{n, k-1}^{2}} .
$$

We investigate Hölderian functional central limit theorems in the two situations in which $\phi_{n}$ tends to one. In the first case, we define $\phi_{n}=e^{\gamma / n}$ ( $\gamma$ is a negative constant), see Phillips [18]; in the second case, $\phi_{n}=1-\gamma_{n} / n, \gamma_{n} \rightarrow \infty$ and $\gamma_{n} / n \rightarrow 0$ as $n \rightarrow \infty$, see Giraitis and Phillips [6]. Note that Markevičiūtè et al. [114] investigated first order nearly nonstationary processes and proved Hölderian limit theorems for the polygonal lines built on observations $y_{n, k}$.

The paper is organized as follows: Section 2 is devoted to some preliminaries and notation; we prove functional limit theorems for the first type model in Section 3, and for the second model in Section 4; Section 5 is devoted to applications. 


\section{WEAK CONVERGENCE IN HÖLDER SPACES}

By $\|f\|_{\infty}$ we denote the uniform norm of $f \in \mathrm{C}[0,1]$. For $\alpha \in[0,1)$ the Hölder space

$$
\mathrm{H}_{\alpha}^{o}[0,1]:=\left\{f \in \mathrm{C}[0,1]: \lim _{\delta \rightarrow 0} \omega_{\alpha}(f, \delta)=0\right\},
$$

endowed with the norm $\|f\|_{\alpha}:=|f(0)|+\omega(f, 1)$, where

$$
\omega_{\alpha}(f, \delta):=\sup _{\substack{s, t \in[0,1] \\ 0<t-s<\delta}} \frac{|f(t)-f(s)|}{|t-s|^{\alpha}},
$$

is a separable Banach space. Throughout the paper, $W=(W(t), t \in[0,1])$ is a standard Brownian motion. By the classical Lévy's result on the modulus of continuity of $W, W \in \mathrm{H}_{\alpha}^{o}[0,1]$ with probability one for every $0 \leqslant \alpha<1 / 2$.

The polygonal line process $W_{n}=\left(W_{n}(t), t \in[0,1], n>0\right)$ built on i.i.d. random variables $\left(\varepsilon_{j}\right)$ is defined by

$$
W_{n}(t)=\sum_{j=1}^{[n t]} \varepsilon_{j}+(n t-[n t]) \varepsilon_{[n t]+1}, \quad W_{n}(0)=0 .
$$

Račkauskas and Suquet [20] proved that for $0<\alpha<1 / 2$ the convergence

$$
n^{-1 / 2} \sigma^{-1} W_{n} \underset{n \rightarrow \infty}{\stackrel{\mathcal{D}}{\longrightarrow}} W \quad \text { in } \mathrm{H}_{\alpha}^{o}[0,1]
$$

holds if and only if

$$
\lim _{t \rightarrow \infty} t^{1 /(1 / 2-\alpha)} P\left(\left|\varepsilon_{1}\right| \geqslant t\right)=0 .
$$

Condition (2.3) provides a precise relation between the strength of the convergence (2.2) and the integrability of summands. Compared with the classical Donsker invariance principle, it shows the price to be paid for functional convergence in a stronger topology. When $\alpha>0$, condition (‥3) implies that $\mathbb{E}\left|\varepsilon_{1}\right|^{p}<\infty$ for $p<(1 / 2-\alpha)^{-1}$ and, in particular, $\mathbb{E} \varepsilon_{1}^{2}<\infty$.

\section{FIRST TYPE MODEL}

3.1. Technical lemmas. To prove the main result for the first type model, we need some technical lemmas.

Lemma 3.1. Let $N_{n}, D_{n}, N, D$ be real-valued random variables with $D_{n}$ and $D$ being nonnegative. Assume that $P(D=0)=0$ and that $\left(N_{n}, D_{n}\right)$ converges in distribution on $\mathbb{R}^{2}$ to $(N, D)$. Define

$$
\Phi_{n}:= \begin{cases}N_{n} / D_{n} & \text { on }\left\{D_{n} \neq 0\right\}, \\ 0 & \text { on }\left\{D_{n}=0\right\} .\end{cases}
$$

Then $\Phi_{n}$ converges in distribution to $N / D$. 
We omit the proof of Lemma B.1. The lemma below is a tool that will help to prove next two lemmas.

LEMMA 3.2. Suppose that the process $\left(y_{n, k}\right)$ is defined by (IL. I) with $\phi_{n}=$ $e^{\gamma / n}, \gamma<0$ and $y_{n, 0}=0$. Let $\left(\varepsilon_{k}\right)$ be i.i.d. random variables with mean zero and satisfying the condition (2.3)). Define

$$
V_{n}(l):=n^{-1 / 2} W_{n}\left(\frac{l-1}{n}\right)+\gamma \int_{0}^{l / n} e^{(l / n-s) \gamma} n^{-1 / 2} W_{n}(s) d s
$$

for $l \leqslant n$. Then

$$
\begin{aligned}
\left|n^{-1 / 2} y_{n, l-1}-V_{n}(l)\right| \leqslant & \left\|n^{-1 / 2} W_{n}\right\|_{\infty} \frac{\gamma^{2} e^{\gamma}}{2 n}+\frac{e^{\gamma}}{n^{\alpha}} \omega_{\alpha}\left(n^{-1 / 2} W_{n}, \frac{1}{n}\right) \\
& +\frac{|2+\gamma| e^{\gamma}}{n}\left\|n^{-1 / 2} W_{n}\right\|_{\infty} .
\end{aligned}
$$

Pro of. Let us define

$$
V_{l, 1}:=n^{-1 / 2} y_{n, l-1}=n^{-1 / 2} \sum_{j=1}^{l-1} e^{(l-1-j) \gamma / n} \varepsilon_{j} .
$$

Noting that $\varepsilon_{l}=W_{n}(l / n)-W_{n}((l-1) / n)$, we can write

$$
\begin{aligned}
y_{n, l-1}= & W_{n}\left(\frac{l-1}{n}\right)+\frac{\gamma}{n} \sum_{j=1}^{l-2} e^{(l-1-j) \gamma / n} W_{n}\left(\frac{j}{n}\right) \\
& +\frac{\gamma^{2} u_{n}}{2 n^{2}} \sum_{j=1}^{l-2} e^{(l-1-j) \gamma / n} W_{n}\left(\frac{j}{n}\right),
\end{aligned}
$$

where $u_{n}$ is defined by

$$
u_{n}=-1+\frac{2 n^{2}}{\gamma^{2}} o\left(\frac{1}{n^{2}}\right),
$$

and $u_{n} \rightarrow-1$ as $n \rightarrow \infty$. Then we define

$$
V_{l, 2}:=n^{-1 / 2} W_{n}\left(\frac{l-1}{n}\right)+\frac{\gamma}{n} \sum_{j=1}^{l-2} e^{(l-1-j) \gamma / n} n^{-1 / 2} W_{n}\left(\frac{j}{n}\right),
$$

and for the approximation error we obtain the bound

$$
\left|V_{l, 2}-V_{l, 1}\right| \leqslant\left\|n^{-1 / 2} W_{n}\right\|_{\infty} \frac{\gamma^{2} e^{\gamma}}{2 n} .
$$


Further, we approximate the Riemann sum by the integral (see (B.1))

$$
V_{n}(l):=n^{-1 / 2} W_{n}\left(\frac{l-1}{n}\right)+\gamma \int_{0}^{l / n} e^{(l / n-s) \gamma} n^{-1 / 2} W_{n}(s) d s .
$$

Now we estimate the error. For any $f \in \mathrm{C}[0,1]$, we have (for details see Markevičiūtė et al. [14])

$$
\left|\frac{1}{n} \sum_{j=1}^{l-2} f\left(\frac{j+j_{0}}{n}\right)-\int_{0}^{l / n} f(s) d s\right| \leqslant \omega_{0}\left(f, \frac{1+j_{0}}{n}\right)+\|f\|_{\infty} \frac{2}{n} .
$$

Moreover,

$$
\text { if } f \in \mathrm{H}_{\alpha}^{o}[0,1], \quad \omega_{0}(f, \delta) \leqslant \omega_{\alpha}(f, \delta) \delta^{\alpha} .
$$

If $f(t)=g(t) h(t)$ with $g$ of class $C^{1}$ and $h \in \mathrm{C}[0,1]$, we get

$$
\omega_{0}(g h, \delta) \leqslant\|g\|_{\infty} \omega_{0}(h, \delta)+\left\|g^{\prime}\right\|_{\infty}\|h\|_{\infty} \delta .
$$

Thus, from (B.4) $-($ (B.6) we obtain the uniform bound

$$
\left|V_{n}(l)-V_{l, 2}\right| \leqslant \frac{e^{\gamma}}{n^{\alpha}} \omega_{\alpha}\left(n^{-1 / 2} W_{n}, \frac{1}{n}\right)+\frac{|2+\gamma| e^{\gamma}}{n}\left\|n^{-1 / 2} W_{n}\right\|_{\infty} .
$$

We will use the following functionals in the proofs of the next two lemmas and the main result of this section:

$$
\begin{gathered}
N(x):=\frac{1}{2}\left(x(1)+\gamma \int_{0}^{1} e^{(1-s) \gamma} x(s) d s\right)^{2} \\
-\gamma \int_{0}^{1}\left(x(r)+\gamma \int_{0}^{r} e^{(r-s) \gamma} x(s) d s\right)^{2} d r-\frac{1}{2}, \\
D(x):=\int_{0}^{1}\left(x(r)+\gamma \int_{0}^{r} e^{(r-s) \gamma} x(s) d s\right)^{2} d r, \\
F(x)(t):=\int_{0}^{t}\left(x(r)+\gamma \int_{0}^{r} e^{(r-s) \gamma} x(s) d s\right) d r, \quad t \in[0,1],
\end{gathered}
$$

for $x \in \mathrm{H}_{\alpha}^{o}[0,1]$.

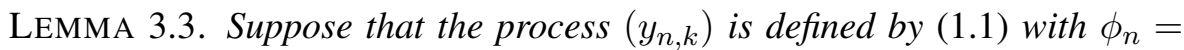
$e^{\gamma / n}, \gamma<0$ and $y_{n, 0}=0$. Let $\left(\varepsilon_{k}\right)$ be i.i.d. random variables with mean zero, $\mathbb{E} \varepsilon_{1}^{2}=1$, and satisfying the condition ([2.3]) for some $\alpha \in(0,1 / 2)$. Define

$$
A_{n, 0}:=n^{-2} \sum_{k=1}^{n} y_{n, k-1}^{2} \text {. }
$$

Then

$$
\left|D\left(n^{-1 / 2} W_{n}\right)-A_{n, 0}\right|=o_{\mathrm{P}}\left(n^{-\alpha}\right) .
$$


P r o o f. Using Lemma B.2, we approximate $A_{n, 0}:=n^{-2} \sum_{k=1}^{n} y_{n, k-1}^{2}$ by

$$
A_{n, 1}:=\frac{1}{n} \sum_{k=1}^{n}\left(n^{-1 / 2} W_{n}\left(\frac{k-1}{n}\right)+\gamma \int_{0}^{k / n} e^{(k / n-s) \gamma} n^{-1 / 2} W_{n}(s) d s\right)^{2} .
$$

The approximation error is bounded by

$$
\begin{aligned}
\left|A_{n, 1}-A_{n, 0}\right| & \leqslant \max _{1 \leqslant k \leqslant n}\left|n^{-1 / 2} y_{n, k-1}-V_{n}(k)\right| \\
& \leqslant \max _{1 \leqslant k \leqslant n}\left|n^{-1 / 2} y_{n, k-1}\right|+\max _{1 \leqslant k \leqslant n}\left|V_{n}(k)\right| .
\end{aligned}
$$

By Lemma 3.2, $\max _{1 \leqslant k \leqslant n}\left|n^{-1 / 2} y_{n, k-1}-V_{n}(k)\right|=o_{\mathrm{P}}\left(n^{-\alpha}\right)$. As $V_{n}(l)$ is the image of $n^{-1 / 2} W_{n}$ by a continuous functional on $\mathrm{H}_{\alpha}^{o}$, we infer from the continuous mapping theorem and Hölderian invariance principle that $\max _{1 \leqslant k \leqslant n}\left|V_{n}(k)\right|$ is stochastically bounded. Also, by [118], $\max _{1 \leqslant k \leqslant n}\left|n^{-1 / 2} y_{n, k-1}\right|$ is stochastically bounded.

Further, $A_{n, 1}$ can be approximated by $A_{n}$, and the bound of approximation error is

$$
\left|A_{n}-A_{n, 1}\right| \leqslant \omega\left(f, \frac{1}{n}\right)
$$

where $f(r):=\left(W_{n}(r)+\gamma \int_{0}^{r} e^{(r-s) \gamma} W_{n}(s) d s\right)^{2}$. Define $g(r):=f^{1 / 2}(r)$. Then

$$
\begin{aligned}
& \text { 12) } \omega\left(f, \frac{1}{n}\right) \leqslant \frac{1}{n^{\alpha}} \omega_{\alpha}\left(f, \frac{1}{n}\right) \leqslant \frac{2}{n^{\alpha}}\|g\|_{\infty} \omega_{\alpha}\left(g, \frac{1}{n}\right) \\
& \leqslant \frac{2}{n^{\alpha}} \cdot\left\|n^{-1 / 2} W_{n}\right\|_{\infty} e^{\gamma}\left(\omega_{\alpha}\left(n^{-1 / 2} W_{n}, \frac{1}{n}\right)+\frac{1}{n^{1-\alpha}} e^{\gamma}\left\|n^{-1 / 2} W_{n}\right\|_{\infty}\right) .
\end{aligned}
$$

So we obtain $\left|A_{n}-A_{n, 0}\right|=o_{\mathrm{P}}\left(n^{-\alpha}\right)$.

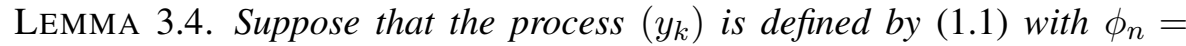
$e^{\gamma / n}, \gamma<0$ and $y_{n, 0}=0$. Let $\left(\varepsilon_{k}\right)$ be i.i.d. random variables with mean zero, $\mathbb{E} \varepsilon_{1}^{2}=1$, and satisfying the condition ([2.3) for some $\alpha \in(0,1 / 2)$. Define

$$
B_{n, 0}:=n^{-1} \sum_{k=1}^{n} \varepsilon_{k} y_{n, k-1}
$$

Then

$$
\left|N\left(n^{-1 / 2} W_{n}\right)-B_{n, 0}\right|=o_{\mathrm{P}}\left(n^{-\alpha}\right) .
$$


Pr o o f. By squaring the equation (ㅍ.J), subtracting $y_{n, k-1}^{2}$ from both sides and summing both sides over $k$, we obtain

$$
y_{n, n}^{2}=\left(e^{2 \gamma / n}-1\right) \sum_{k=1}^{n} y_{n, k-1}^{2}+2 e^{\gamma / n} \sum_{k=1}^{n} y_{n, k-1} \varepsilon_{k}+\sum_{k=1}^{n} \varepsilon_{k}^{2} .
$$

Then multiplying everything by $n^{-1}$, we get

$$
\begin{aligned}
B_{n, 1}: & =2 n^{-1} \sum_{k=1}^{n} y_{n, k-1} \varepsilon_{k} \\
& =\frac{1}{e^{\gamma / n}}\left(n^{-1} y_{n, n}^{2}-\frac{2 \gamma}{n^{2}} \sum_{k=1}^{n} y_{n, k-1}^{2}-\frac{1}{n} \sum_{k=1}^{n} \varepsilon_{k}^{2}-\frac{\gamma^{2} u_{n}}{n^{3}} \sum_{k=1}^{n} y_{n, k-1}^{2}\right),
\end{aligned}
$$

where $u_{n} \rightarrow-1$ as $n \rightarrow \infty$. Further, we can approximate $B_{n, 1}$ by

$$
B_{n, 2}:=\frac{1}{e^{\gamma / n}}\left(n^{-1} y_{n, n}^{2}-\frac{2 \gamma}{n^{2}} \sum_{k=1}^{n} y_{n, k-1}^{2}-\frac{1}{n} \sum_{k=1}^{n} \varepsilon_{k}^{2}\right),
$$

and the bound of the approximation error is

$$
\left|B_{n, 2}-B_{n, 1}\right| \leqslant \frac{\gamma^{2}}{n}\left|\frac{1}{n^{2}} \sum_{k=1}^{n} y_{n, k-1}^{2}\right| \underset{n \rightarrow \infty}{\stackrel{\mathrm{P}}{\longrightarrow}} 0
$$

because, by [18], $\left|n^{-2} \sum_{k=1}^{n} y_{n, k-1}^{2}\right|$ is stochastically bounded on $\mathbb{R}$ and $\gamma^{2} / n \rightarrow 0$ as $n \rightarrow \infty$. Further, we can approximate $B_{n, 2}$ by

$$
B_{n, 3}:=\frac{1}{e^{\gamma / n}}\left(n^{-1} y_{n, n}^{2}-\frac{2 \gamma}{n^{2}} \sum_{k=1}^{n} y_{n, k-1}^{2}-1\right) .
$$

In this case, for the approximation error we have

$$
\left|B_{n, 3}-B_{n, 2}\right| \leqslant\left|\frac{1}{n} \sum_{k=1}^{n} \varepsilon_{k}^{2}-1\right| \underset{n \rightarrow \infty}{\stackrel{\mathrm{P}}{\longrightarrow}} 0
$$

by the weak law of large numbers since $\mathbb{E} \varepsilon_{0}^{2}=1$. Next, we approximate $B_{n, 3}$ by

$$
B_{n, 4}:=n^{-1} y_{n, n}^{2}-\frac{2 \gamma}{n^{2}} \sum_{k=1}^{n} y_{n, k-1}^{2}-1
$$

As $\left|n^{-1} y_{n, n}^{2}-\left(2 \gamma / n^{2}\right) \sum_{k=1}^{n} y_{n, k-1}^{2}-1\right|$ is stochastically bounded by Lemma 1 in [118], we obtain

$$
\left|B_{n, 4}-B_{n, 3}\right|=\left|n^{-1} y_{n, n}^{2}-\frac{2 \gamma}{n^{2}} \sum_{k=1}^{n} y_{n, k-1}^{2}-1\right| \cdot\left|1-\frac{1}{e^{\gamma / n}}\right| \underset{n \rightarrow \infty}{\stackrel{\mathrm{P}}{\longrightarrow}} 0 .
$$


Finally, put $g(r)=W_{n}(r)+\gamma \int_{0}^{r} e^{(r-s) \gamma} W_{n}(s) d s$ and define

$$
B_{n}=\frac{1}{2}(g(1))^{2}-\gamma \int_{0}^{1}(g(r))^{2} d r-\frac{1}{2}
$$

Then, using Lemma 3.2, we obtain

$$
\begin{aligned}
\left|B_{n}-B_{n, 4}\right| \leqslant & \frac{1}{2}\left|\left(n^{-1 / 2} y_{n, n}\right)^{2}-\left(W_{n}(1)+\gamma \int_{0}^{1} e^{(1-s) \gamma} W_{n}(s) d s\right)^{2}\right| \\
& +\gamma\left|\frac{1}{n^{2}} \sum_{k=1}^{n} y_{n, k-1}^{2}-\int_{0}^{1}\left(W_{n}(r)+\gamma \int_{0}^{r} e^{(r-s) \gamma} W_{n}(s) d s\right)^{2} d r\right| .
\end{aligned}
$$

Further, the first summand is bounded by (B.TI) and the second one is bounded by (B.11) and (B.12).

3.2. Invariance principle. For the process $\widehat{W}_{n}$ defined by (L.2) we prove an invariance principle under the necessary and sufficient condition ([2.3)).

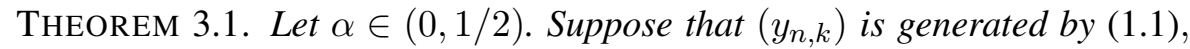
$\phi_{n}=e^{\gamma / n}$, and $\gamma<0$ is a constant. Assume that $\left(\varepsilon_{k}\right)$ are i.i.d. random variables with $\mathbb{E} \varepsilon_{0}=0, \mathbb{E} \varepsilon_{0}^{2}=\sigma^{2}$ and $y_{n, 0}=0$. Then

$$
n^{-1 / 2} \sigma^{-1} \widehat{W}_{n} \underset{n \rightarrow \infty}{\stackrel{\mathcal{D}}{\longrightarrow}} W-A^{-1} B J \quad \text { in } \mathrm{H}_{\alpha}^{o}[0,1]
$$

if and only if condition ([2.3) holds. Here $A=\int_{0}^{1} U_{\gamma}^{2}(t) d t, B=\int_{0}^{1} U_{\gamma}(t) d W(t)$, and $J(t)$ is an integrated Ornstein-Uhlenbeck process defined by

$$
J(t):=\int_{0}^{t} U_{\gamma}(s) d s,
$$

where $U_{\gamma}(r)$ is an Ornstein-Uhlenbeck process,

$$
U_{\gamma}(r):=\int_{0}^{r} e^{(r-s) \gamma} d W(s), \quad U_{\gamma}(0)=0 .
$$

REMARK 3.1. If variance is unknown, then by Slutsky's theorem it can be replaced in (3.14), via Theorem 1 in Phillips [18], by its estimator defined by

$$
\widehat{\sigma}^{2}:=\frac{1}{n} \sum_{k=1}^{n} \widehat{\varepsilon}_{k}^{2}
$$

Proof of Theorem 3.1. Without loss of generality we assume that $\sigma^{2}=1$. 
To prove the sufficiency, at first, we express $\widehat{W}_{n}$ in terms of $W_{n}$ and the polygonal line process $S_{n}$ built on observations $y_{k}$ :

$$
n^{-1 / 2} \widehat{W}_{n}=n^{-1 / 2} W_{n}-\frac{n^{-1} \sum_{k=1}^{n} \varepsilon_{k} y_{n, k-1}}{n^{-2} \sum_{k=1}^{n} y_{n, k-1}^{2}} \cdot n^{-3 / 2} S_{n},
$$

where $S_{n}=\left(S_{n}(t), t \in[0,1], n>0\right)$, and

$$
S_{n}(t)=\sum_{j=1}^{[n t]} y_{n, j-1}+(n r-[n t]) y_{n,[n t]}, \quad S_{n}(0)=0 .
$$

Next, using the definition of $U_{\gamma}$, we obtain, by Itô's formula,

$$
\int_{0}^{1} U_{\gamma}(r) d W(r)=\frac{1}{2}\left(U_{\gamma}^{2}(1)-1-2 \gamma \int_{0}^{1} U_{\gamma}^{2}(r) d r\right) .
$$

Further, define the operator $T$,

$$
T(W):=W-A^{-1} B J,
$$

so that

$$
T: \mathrm{H}_{\alpha}^{o}[0,1] \rightarrow \mathrm{H}_{\alpha}^{o}[0,1], \quad T(x):=x-\frac{N(x)}{D(x)} F(x),
$$

where $N(x), D(x)$ and $F(x)$ are defined by (B.7), (B.8) and (B.9), respectively. It is obvious that the domain of the operator $T$ is

$$
H_{T}:=\left\{x \in \mathrm{H}_{\alpha}^{o}[0,1]: D(x) \neq 0\right\} .
$$

Further note that $H_{T}$ is the Hölder space without the zero functions. Indeed, from the equations $D(x)=0$, recalling that $x$ is a continuous function on $[0,1]$, we obtain for every $r \in[0,1]$

$$
x(r)+\gamma \int_{0}^{r} e^{(r-s) \gamma} x(s) d s=0 .
$$

Thus, any continuous solution $x$ of $D(x)=0$ satisfies

$$
x(r)=-\gamma e^{r \gamma} \int_{0}^{r} e^{-s \gamma} x(s) d s .
$$


Further, from the continuity of $x$ it follows that the right-hand side of (B.2I) is obviously differentiable; consequently, $x$ is itself differentiable, and for all $r \in(0,1)$ we obtain $x^{\prime}(r)=0$. This implies that $x$ is a constant on $[0,1]$ (it is continuous at zero and at one). Let $r$ tend to zero in (B.2II). Then by the continuity of $x$ we obtain $x(0)=0$, and since $x$ is constant, $x(r)=0$ for every $r \in[0,1]$. Thus we obtain

$$
\mathbb{P}\left(W \in \mathrm{H}_{\alpha}^{o}[0,1] \backslash H_{T}\right)=\mathbb{P}(W=0)=0 .
$$

Next, we obtain the convergence (B.14) by proving that

(a) $T$ is a continuous operator on $H_{T}$, and $\mathbb{P}\left(W \in \mathrm{H}_{\alpha}^{o}[0,1] \backslash H_{T}\right)=0$;

(b) $\left\|n^{-1 / 2} \widehat{W}_{n}-T\left(n^{-1 / 2} W_{n}\right)\right\|_{\alpha} \underset{n \rightarrow \infty}{\stackrel{\mathrm{P}}{\longrightarrow}} 0$.

We start with the continuity of $T$. The operator $T$ is the difference of two operators. The first one is the identity on $\mathrm{H}_{\alpha}^{o}[0,1]$, obviously continuous. The second one is

$$
\widetilde{T}(x):=\frac{N(x)}{D(x)} \cdot F(x), \quad x \in H_{T} .
$$

First we show that $N: \mathrm{H}_{\alpha}^{o}[0,1] \rightarrow \mathbb{R}$ and $D: \mathrm{H}_{\alpha}^{o}[0,1] \rightarrow \mathbb{R}$ are continuous. Let us check first the continuity of $D$. By the triangular inequality of $L_{2}$-norm applied to the function $f(x)(r)=x(r)+\gamma \int_{0}^{r} e^{(r-s) \gamma} x(s) d s$, we have

$$
\begin{aligned}
\mid D^{1 / 2}(x) & -D^{1 / 2}(y)|=|\left(\int_{0}^{1}(f(x)(r))^{2} d r\right)^{1 / 2}-\left(\int_{0}^{1}(f(y)(r))^{2} d r\right)^{1 / 2} \mid \\
& \leqslant\left|\left(\int_{0}^{1}\left(|x(r)-y(r)|+\gamma \int_{0}^{r} e^{(r-s) \gamma}|x(s)-y(s)| d s\right)^{2} d r\right)^{1 / 2}\right| \\
& \leqslant\|x-y\|_{\infty}\left(\frac{1}{2 \gamma}\left(e^{2 \gamma}-1\right)\right)^{1 / 2} .
\end{aligned}
$$

Since $\|\cdot\|_{\infty} \leqslant\|\cdot\|_{\alpha}$, we obtain

$$
\left|D^{1 / 2}(x)-D^{1 / 2}(y)\right| \leqslant\left(\frac{1}{2 \gamma}\left(e^{2 \gamma}-1\right)\right)^{1 / 2}\|x-y\|_{\alpha} .
$$

This implies that $D^{1 / 2}$ is continuous on $\mathrm{H}_{\alpha}^{o}[0,1]$, and so is $D$. Using the same arguments, we obtain the continuity of $N$ on $\mathrm{H}_{\alpha}^{o}[0,1]$.

Thus, the ratio $N / D$ is continuous as the ratio of two continuous functions except on the subset of $\mathrm{H}_{\alpha}^{o}[0,1]$, where $D(x)=0$, that is, at the null function on $[0,1]$.

As $F$ is linear, it is enough to show its continuity at zero. Recall that

$$
\|F(x)\|_{\alpha}=|F(x)(0)|+\sup _{0 \leqslant t^{\prime}<t \leqslant 1} \frac{\left|F(x)(t)-F(x)\left(t^{\prime}\right)\right|}{\left|t-t^{\prime}\right|^{\alpha}} .
$$


Noting $\|x\|_{\infty} \leqslant\|x\|_{\alpha}$, we see that

$$
\begin{aligned}
\left|F(x)(t)-F(x)\left(t^{\prime}\right)\right| & =\left|\int_{t^{\prime}}^{t}\left(x(r)+\gamma \int_{0}^{r} e^{(r-s) \gamma} x(s) d s\right) d r\right| \\
& \leqslant\left(1+\gamma e^{\gamma}\right)\|x\|_{\alpha}\left|t-t^{\prime}\right| .
\end{aligned}
$$

Since $F(x)(0)=0$, we obtain

$$
\|F(x)\|_{\alpha} \leqslant\left(1+\gamma e^{\gamma}\right)\|x\|_{\alpha}
$$

which gives the continuity of $F$.

The continuity of $\widetilde{T}$ on $H_{T}$ follows easily from the continuity of $N, D$ and $F$. Finally, the operator $T$ is continuous on $H_{T}$ as the difference of two continuous operators.

As the operator $T$ is continuous on $H_{T}$ and $\mathbb{P}(W=0)=0$, also the Hölderian invariance principle holds (see [21] ); then we have

$$
T\left(n^{-1 / 2} W_{n}\right) \underset{n \rightarrow \infty}{\stackrel{\mathcal{D}}{\longrightarrow}} T(W)=W-A^{-1} B J \quad \text { in } \mathrm{H}_{\alpha}^{o}[0,1]
$$

by the continuous mapping theorem (for details see [3], Theorem 5.1).

By Lemmas 3.3 and 3.4 we have

$$
\begin{aligned}
n^{-1} \sum_{k=1}^{n} \varepsilon_{k} y_{n, k-1} & :=N\left(n^{-1 / 2} W_{n}\right)+R_{n}, \\
n^{-2} \sum_{k=1}^{n} y_{n, k-1}^{2} & :=D\left(n^{-1 / 2} W_{n}\right)+\widetilde{R}_{n},
\end{aligned}
$$

where $R_{n}=o_{\mathrm{P}}\left(n^{-\alpha}\right)$ and $\widetilde{R}_{n}=o_{\mathrm{P}}\left(n^{-\alpha}\right)$. We have also

$$
n^{-3 / 2} S_{n}(t)=F\left(n^{-1 / 2} W_{n}\right)(t)+\widetilde{\widetilde{R}}_{n}, \quad t \in[0,1],
$$

where $\widetilde{\widetilde{R}}_{n}=o_{\mathrm{P}}\left(n^{-\alpha}\right)$ (for details see the proof of Theorem 1 in [14]).

Further, we can express $\widehat{W}_{n}$ as

$$
n^{-1 / 2} \widehat{W}_{n}=n^{-1 / 2} W_{n}-\frac{N\left(n^{-1 / 2} W_{n}\right)+R_{n}}{D\left(n^{-1 / 2} W_{n}\right)+\widetilde{R}_{n}} \cdot\left(F\left(n^{-1 / 2} W_{n}\right)(t)+\widetilde{\widetilde{R}}_{n}\right),
$$

and we obtain

$$
\begin{aligned}
\left\|n^{-1 / 2} \widehat{W}_{n}-T\left(n^{-1 / 2} W_{n}\right)\right\|_{\alpha} \\
\leqslant\left|\frac{N\left(n^{-1 / 2} W_{n}\right)+R_{n}}{D\left(n^{-1 / 2} W_{n}\right)+\widetilde{R}_{n}}-\frac{N\left(n^{-1 / 2} W_{n}\right)}{D\left(n^{-1 / 2} W_{n}\right)}\right|\left\|F\left(n^{-1 / 2} W_{n}\right)+\widetilde{\widetilde{R}}_{n}\right\|_{\alpha} \\
\quad+\left|\frac{N\left(n^{-1 / 2} W_{n}\right)}{D\left(n^{-1 / 2} W_{n}\right)}\right|\left\|\widetilde{\widetilde{R}}_{n}\right\|_{\alpha} .
\end{aligned}
$$


Let us to introduce the random variables $\Phi_{n}$ and $\widetilde{\Phi}_{n}$ defined by

$$
\begin{gathered}
\Phi_{n}:= \begin{cases}\frac{N\left(n^{-1 / 2} W_{n}\right)}{D\left(n^{-1 / 2} W_{n}\right)} & \text { on }\left\{D\left(n^{-1 / 2} W_{n}\right) \neq 0\right\}, \\
0 & \text { on }\left\{D\left(n^{-1 / 2} W_{n}\right)=0\right\},\end{cases} \\
\widetilde{\Phi}_{n}:= \begin{cases}\frac{N\left(n^{-1 / 2} W_{n}\right)+R_{n}}{D\left(n^{-1 / 2} W_{n}\right)+\widetilde{R}_{n}} & \text { on }\left\{D\left(n^{-1 / 2} W_{n}\right)+\widetilde{R}_{n} \neq 0\right\}, \\
0 & \text { on }\left\{D\left(n^{-1 / 2} W_{n}\right)+\widetilde{R}_{n}=0\right\} .\end{cases}
\end{gathered}
$$

Coming back to the decomposition of $n^{1 / 2} \widehat{W}_{n}$ and modifying the definition of $T\left(n^{-1 / 2} W_{n}\right)$ as

$$
T\left(n^{-1 / 2} W_{n}\right)=n^{-1 / 2} W_{n}-\Phi_{n} F\left(n^{-1 / 2} W_{n}\right)
$$

(for that purpose it suffices to define $T(0):=0$ ), we can rewrite the estimate of $\left\|n^{1 / 2} \widehat{W}_{n}-T\left(n^{-1 / 2} W_{n}\right)\right\|_{\alpha}$ as

$\left\|n^{1 / 2} \widehat{W}_{n}-T\left(n^{-1 / 2} W_{n}\right)\right\|_{\alpha} \leqslant\left|\Phi_{n}-\widetilde{\Phi}_{n}\right|\left\|F\left(n^{-1 / 2} W_{n}\right)+\widetilde{\widetilde{R}}_{n}\right\|_{\alpha}+\left|\Phi_{n}\right|\left\|\widetilde{\widetilde{R}}_{n}\right\|_{\alpha}$.

By continuous mapping, $\left(N\left(n^{-1 / 2} W_{n}\right), D\left(n^{-1 / 2} W_{n}\right)\right)$ converges in distribution in $\mathbb{R}^{2}$ to $(N(W), D(W))=(B, A)$. In view of $P(D(W)=0)=0$, Lemma $B .1$ gives us the convergence in distribution of $\Phi_{n}$ to $B / A$, and, in particular, $\Phi_{n}$ is stochastically bounded.

Since $\left\|\widetilde{\widetilde{R}}_{n}\right\|_{\alpha}$ converges to zero in probability and $\left\|F\left(n^{-1 / 2} W_{n}\right)\right\|_{\alpha}$ is stochastically bounded, it remains only to check that $\left|\Phi_{n}-\widetilde{\Phi}_{n}\right|$ converges to zero in probability.

Note that

$$
\left|\Phi_{n}-\widetilde{\Phi}_{n}\right| \leqslant \frac{\left|R_{n}\right|}{\left|D\left(n^{-1 / 2} W_{n}\right)+\widetilde{R}_{n}\right|}+\left|\frac{N\left(n^{-1 / 2} W_{n}\right)}{D\left(n^{-1 / 2} W_{n}\right)}\right| \cdot \frac{\left|\widetilde{R}_{n}\right|}{\left|D\left(n^{-1 / 2} W_{n}\right)+\widetilde{R}_{n}\right|} .
$$

So the problem reduces to proving that

$$
\frac{\left|R_{n}\right|}{\left|D\left(n^{-1 / 2} W_{n}\right)+\widetilde{R}_{n}\right|} \underset{n \rightarrow \infty}{\stackrel{\mathrm{P}}{\longrightarrow}} 0 \text { and } \quad \frac{\left|\widetilde{R}_{n}\right|}{\left|D\left(n^{-1 / 2} W_{n}\right)+\widetilde{R}_{n}\right|} \underset{n \rightarrow \infty}{\stackrel{\mathrm{P}}{\longrightarrow}} 0 .
$$

But these two convergences can be easily checked, so finally the convergence (B.14) is established.

The next step is to prove the necessity. By (B.14), the sequence $\left(n^{-1 / 2} \widehat{W}_{n}\right)$ is tight on $\mathrm{H}_{\alpha}^{o}[0,1]$, and this implies that, for every $\epsilon>0$,

$$
\lim _{\delta \rightarrow 0} \sup _{n \geqslant 1} P\left(\omega_{\alpha}\left(n^{-1 / 2} \widehat{W}_{n}, \delta\right)>\epsilon\right)=0
$$


see, e.g., Theorem 13 in [24]. This clearly entails that $\omega_{\alpha}\left(n^{-1 / 2} \widehat{W}_{n}, 1 / n\right) \underset{n \rightarrow \infty}{\stackrel{\mathrm{P}}{\longrightarrow}} 0$. Since

$$
\begin{aligned}
\frac{n^{-1 / 2} \max _{1 \leqslant k \leqslant n}\left|\widehat{\varepsilon}_{k}\right|}{n^{-\alpha}} & =n^{-1 / 2+\alpha} \max _{1 \leqslant k \leqslant n}\left|\widehat{W}_{n}\left(\frac{k}{n}\right)-\widehat{W}_{n}\left(\frac{k-1}{n}\right)\right| \\
& \leqslant \omega_{\alpha}\left(n^{-1 / 2} \widehat{W}_{n}, \frac{1}{n}\right),
\end{aligned}
$$

we obtain $n^{-1 / 2} \max _{1 \leqslant k \leqslant n}\left|\widehat{\varepsilon}_{k}\right| \underset{n \rightarrow \infty}{\stackrel{\mathrm{P}}{\longrightarrow}} 0$.

Next decompose $\widehat{\varepsilon}_{k}=\varepsilon_{k}-\left(\widehat{\phi_{n}}-\phi_{n}\right) y_{n, k-1}$. Denote by $y_{n,[n \bullet]}$ the step process $\left(y_{n,[n t]}, t \in[0,1]\right)$. Recall that, by [118], Lemma 1, part (a), $n^{-1 / 2} y_{n,[n \bullet]}$ converges in distribution in $\mathrm{D}[0,1]$ to an Ornstein-Uhlenbeck process. As the supremum norm of such a step process is obviously reached at one of the points $t=k / n$, $0 \leqslant k \leqslant n$, this convergence implies the stochastic boundedness of

$$
\max _{1 \leqslant k \leqslant n}\left|n^{-1 / 2} y_{n, k-1}\right|=\left\|n^{-1 / 2} y_{n,[n \bullet]}\right\|_{\infty} .
$$

Notice that

$$
\begin{aligned}
& n^{\alpha-1 / 2} \max _{1 \leqslant k \leqslant n}\left|\left(\widehat{\phi_{n}}-\phi_{n}\right) y_{n, k-1}\right| \\
& \quad \leqslant n^{\alpha-1}\left|n\left(\widehat{\phi_{n}}-\phi_{n}\right)\right| \max _{1 \leqslant k \leqslant n}\left|n^{-1 / 2} y_{n, k-1}\right| \underset{n \rightarrow \infty}{\mathrm{P}} 0,
\end{aligned}
$$

because by [18] (Theorem 1, part (a)) $\left|n\left(\widehat{\phi_{n}}-\phi_{n}\right)\right|$ is also stochastically bounded. It follows then that

$$
n^{\alpha-1 / 2} \max _{1 \leqslant k \leqslant n}\left|\varepsilon_{k}\right| \underset{n \rightarrow \infty}{\stackrel{\mathrm{P}}{\longrightarrow}} 0,
$$

which gives the condition ([2.3]) by the independence of $\left(\varepsilon_{k}\right)$.

\section{SECOND TYPE MODEL}

For the second type model we obtain the result of the convergence of $n^{-1 / 2} \widehat{W}_{n}$ to Wiener process in $\mathrm{H}_{\beta}^{o}[0,1]$ for $0<\beta \leqslant \alpha$, assuming additionally some rate of divergence for $\gamma_{n}$.

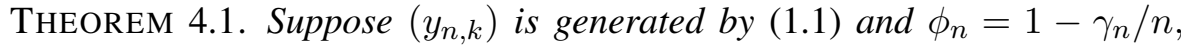
where $\left(\gamma_{n}\right)$ is a sequence of nonnegative numbers, $\gamma_{n} \rightarrow \infty$ and $\gamma_{n} / n \rightarrow 0$ as $n \rightarrow \infty$. Assume also that the innovations $\left(\varepsilon_{k}\right)$ are i.i.d. with mean zero, $\mathbb{E} \varepsilon_{1}^{2}=$ $\sigma^{2}$, and satisfy condition (2.3): $\lim _{t \rightarrow \infty} t^{p} \mathbb{P}\left(\left|\varepsilon_{0}\right|>t\right)=0$ for some $p>2$. Put $\alpha=1 / 2-1 / p$. Then, for $0<\beta \leqslant \alpha$,

$$
n^{-1 / 2} \sigma^{-1} \widehat{W}_{n} \underset{n \rightarrow \infty}{\stackrel{\mathcal{D}}{\longrightarrow}} W \quad \text { in } \mathrm{H}_{\beta}^{o}[0,1]
$$


if $y_{n, 0}=0$ and

$$
\liminf _{n \rightarrow \infty} \gamma_{n} n^{-2 \beta /(1+2 \alpha)}>0 .
$$

REMARK 4.1. If variance is unknown, by Slutsky's theorem it can be replaced in (4.1) by its estimator defined by (B.T7) via Lemma 4.1] below.

Pro of of The or e m 4.1. Condition ([2.3) (see [21]) gives the weak convergence of $W_{n}$, defined by (R.I), to the standard Brownian motion in the space $\mathrm{H}_{\alpha}^{o}[0,1]$. By continuous embedding of Hölder spaces, the same convergence remains true in $H_{\beta}^{0}[0,1]$ for $0<\beta \leqslant \alpha$. Therefore, to obtain (4.]) it suffices to prove that

$$
\Delta_{n, \beta}:=\left\|n^{-1 / 2} \widehat{W}_{n}-n^{-1 / 2} W_{n}\right\|_{\beta} \underset{n \rightarrow \infty}{\stackrel{\mathrm{P}}{\longrightarrow}} 0 .
$$

We first establish the useful inequality

$$
\left\|S_{n}\right\|_{\beta} \leqslant \frac{n}{\gamma_{n}}\left[\left\|W_{n}\right\|_{\beta}+2 n^{\beta} \max _{1 \leqslant k \leqslant n}\left|y_{n, k}\right|\right],
$$

where $S_{n}$ is defined by (B.T). We have, for $1 \leqslant j<k \leqslant n$,

$$
S_{n}(k / n)-S_{n}(j / n)=\left(1-\phi_{n}\right)^{-1}\left(W_{n}(k / n)-W_{n}(j / n)-y_{n, k}+y_{n, j}\right) .
$$

Recalling that the Hölder norm of a polygonal line is reached at some pair of vertices (see Lemma A. 2 in [14]) and that $S_{n}(0)=0$, we have

$$
\begin{aligned}
\left\|S_{n}\right\|_{\beta} & =\max _{1 \leqslant j<k \leqslant n} \frac{\left|\left(1-\phi_{n}\right)^{-1}\left(W_{n}(k / n)-W_{n}(j / n)-y_{n, k}+y_{n, j}\right)\right|}{|k / n-j / n|^{\beta}} \\
& \leqslant \frac{n}{\gamma_{n}}\left[\max _{1 \leqslant j<k \leqslant n} \frac{\left|W_{n}(k / n)-W_{n}(j / n)\right|}{|k / n-j / n|^{\beta}}+\max _{1 \leqslant j<k \leqslant n} \frac{\left|y_{n, k}-y_{n, j}\right|}{|k / n-j / n|^{\beta}}\right] \\
& =\frac{n}{\gamma_{n}}\left[\left\|W_{n}\right\|_{\beta}+\max _{1 \leqslant j<k \leqslant n} \frac{\left|y_{n, k}-y_{n, j}\right|}{|k / n-j / n|^{\beta}}\right] .
\end{aligned}
$$

This leads to (4.3) via the elementary estimate

$$
\max _{1 \leqslant j<k \leqslant n} \frac{\left|y_{n, k}-y_{n, j}\right|}{|k / n-j / n|^{\beta}} \leqslant 2 n^{\beta} \max _{1 \leqslant k \leqslant n}\left|y_{n, k}\right| .
$$

Note that $\widehat{W}_{n}=W_{n}+\left(\phi_{n}-\widehat{\phi}_{n}\right) S_{n}$, see (3.18)), thus we have

$$
\Delta_{n, \beta}=n^{-1 / 2}\left|\phi_{n}-\widehat{\phi}_{n}\right|\left\|S_{n}\right\|_{\beta} .
$$

By the results in [6], there is a positive random variable $M$ not depending on $n$, such that $\left|\phi_{n}-\widehat{\phi}_{n}\right| \leqslant M n^{-1} \gamma_{n}^{1 / 2}$, so using (4.3)), we can bound $\Delta_{n, \beta}$ as follows:

$$
\Delta_{n, \beta} \leqslant M n^{-1 / 2} \gamma_{n}^{-1 / 2}\left(\left\|W_{n}\right\|_{\beta}+2 n^{\beta} \max _{1 \leqslant k \leqslant n}\left|y_{n, k}\right|\right) .
$$


As $n^{-1 / 2}\left\|W_{n}\right\|_{\beta}$ is stochastically bounded, the proof of the theorem is finally reduced to checking that

$$
n^{-1 / 2+\beta} \gamma_{n}^{-1 / 2} \max _{1 \leqslant k \leqslant n}\left|y_{n, k}\right| \underset{n \rightarrow \infty}{\stackrel{\mathrm{P}}{\longrightarrow}} 0 .
$$

By Lemma 1 in [14], $\max _{1 \leqslant k \leqslant n}\left|y_{n, k}\right|=o_{P}\left(n^{1 / 2} \gamma_{n}^{-\alpha}\right)$, so the above convergence holds provided that

$$
\limsup _{n \rightarrow \infty} \frac{n^{\beta}}{\gamma_{n}^{1 / 2+\alpha}}<\infty,
$$

which is equivalent to assumption (4.2).

Next we show that, for the second type model defined by (‥J), the estimate of variance is consistent.

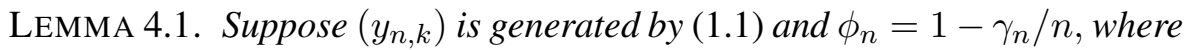
$\gamma_{n}$ is a sequence of nonnegative numbers, $\gamma_{n} / n \rightarrow 0$ and $\gamma_{n} \rightarrow \infty$ as $n \rightarrow \infty$. Assume also that the innovations $\left(\varepsilon_{k}\right)$ are i.i.d. random variables with $\mathbb{E} \varepsilon_{k}=0$, $\mathbb{E} \varepsilon_{k}^{2}=\sigma^{2}$. The variance estimator $\widehat{\sigma}^{2}$ is defined by (B.17). Then

$$
\widehat{\sigma}^{2} \underset{n \rightarrow \infty}{\stackrel{\mathrm{P}}{\longrightarrow}} \sigma^{2} .
$$

P r o o f. We can rearrange (B.17), using ([L.3), in the following way:

$$
\begin{aligned}
\widehat{\sigma}^{2}=\frac{1}{n} \sum_{k=1}^{n} \widehat{\varepsilon}_{k}^{2}= & \frac{1}{n} \sum_{k=1}^{n} \varepsilon_{k}^{2}-\frac{2}{n}\left(\widehat{\phi}_{n}-\phi_{n}\right) \sum_{k=1}^{n} \varepsilon_{k} y_{n, k-1} \\
& +\frac{1}{n}\left(\widehat{\phi_{n}}-\phi_{n}\right)^{2} \sum_{k=1}^{n} y_{n, k-1}^{2} .
\end{aligned}
$$

By the weak law of large numbers we have

$$
\frac{1}{n} \sum_{k=1}^{n} \varepsilon_{k}^{2} \underset{n \rightarrow \infty}{\stackrel{\mathrm{P}}{\longrightarrow}} \sigma^{2}
$$

Using (5) and (10) of Giraitis and Phillips [6] for $\frac{1}{n}\left(\widehat{\phi_{n}}-\phi_{n}\right)^{2} \sum_{k=1}^{n} y_{n, k-1}^{2}$, we obtain

$$
\frac{1}{n}\left(\widehat{\phi_{n}}-\phi_{n}\right)^{2} \sum_{k=1}^{n} y_{n, k-1}^{2} \underset{n \rightarrow \infty}{\stackrel{\mathrm{P}}{\longrightarrow}} 0 .
$$

Also, for $\frac{2}{n}\left(\widehat{\phi}_{n}-\phi_{n}\right) \sum_{k=1}^{n} \varepsilon_{k} y_{n, k-1}$, by (5) and (9) in Giraitis and Phillips [6], we find

$$
\frac{2}{n}\left(\widehat{\phi}_{n}-\phi_{n}\right) \sum_{k=1}^{n} \varepsilon_{k} y_{n, k-1} \underset{n \rightarrow \infty}{\stackrel{\mathrm{P}}{\longrightarrow}} 0 .
$$

Thus (4.6)-(4.8) give us (4.5)). 


\section{APPLICATIONS}

The Hölderian limit theorems provide applications via continuous mapping. To be more precise, we will formulate two general corollaries.

COROLLARY 5.1. Under the conditions of Theorem B.1, for any continuous function $F: \mathrm{H}_{\alpha}^{o}[0,1] \mapsto \mathbb{R}, \alpha \in(0,1 / 2)$,

$$
F\left(n^{-1 / 2} \sigma^{-1} \widehat{W}_{n}\right) \underset{n \rightarrow \infty}{\stackrel{\mathcal{D}}{\longrightarrow}} F\left(W-A^{-1} B J\right) .
$$

COROLlARY 5.2. Under the conditions of Theorem 1.1, for any continuous function $\widetilde{F}: \mathrm{H}_{\beta}^{o}[0,1] \mapsto \mathbb{R}, \beta \in(0,1 / 2-1 / p), p>2$,

$$
\widetilde{F}\left(n^{-1 / 2} \sigma^{-1} \widehat{W}_{n}\right) \underset{n \rightarrow \infty}{\stackrel{\mathcal{D}}{\longrightarrow}} \widetilde{F}(W) .
$$

5.1. Epidemic change detection. In this section we give an application of functional limit theorems to the epidemic type change problem. The epidemic change in parameter $\theta$ is described as the change at some unknown time or location such that $\theta_{1}=\ldots=\theta_{k^{*}}=\theta_{m^{*}+1}=\theta_{n}=\theta_{0}$ and $\theta_{k^{*}+1}=\ldots=\theta_{m^{*}}=\theta$. That is, at some point of time or location the value of the unknown parameter changes, but after a certain period it returns to an initial value. Epidemic change detection is a widely investigated question. We refer to Levin and Kline [13], Commenges et al. [5], Broemeling and Tsurumu [4], Gombay [7], Avery and Henderson [1]], etc., for more information. The Hölderian framework for epidemic change was introduced by Račkauskas and Suquet [21] for i.i.d. random variables. They showed that the Hölderian weighting allows us to detect epidemics shorter than $\sqrt{n}$.

We implement this setting for the innovations of the first order nearly nonstationary autoregressive process. Assume we are given a sample $y_{n, 1}, \ldots, y_{n, n}$ for a fixed $n$, generated from the first order autoregressive process

$$
y_{n, k}=\phi_{n} y_{n, k-1}+\varepsilon_{k}+a_{n, k}, \quad k=1, \ldots, n, n \geqslant 1, \quad y_{n, 0}=0,
$$

where $\phi_{n}=e^{\gamma / n}, \gamma<0$ is a constant, or $\phi_{n}=1-\gamma_{n} / n$, where $\left(\gamma_{n}\right)$ is a sequence of nonnegative numbers, $\gamma_{n} \rightarrow \infty$ and $\gamma_{n} / n \rightarrow 0$ as $n \rightarrow \infty$. The innovations $\left(\varepsilon_{k}, k \leqslant n\right)$ are unobservable, centered, square integrable random variables.

The aim is to propose tests for the null hypothesis

$$
H_{0}: a_{n, 1}=\ldots=a_{n, n}=0
$$

against the epidemic alternative

$$
\begin{aligned}
& H_{A}: \text { there exist } 0 \leqslant k_{n}^{*}<n, \text { and } 1 \leqslant m_{n}^{*} \leqslant n \text { such that } \\
& \qquad a_{n, k}=a_{n} \neq 0 \text { for } k \in \mathbb{I}_{n}^{*}, \text { whereas } a_{n, k}=0 \text { for } k \notin \mathbb{I}_{n}^{*},
\end{aligned}
$$


where $\mathbb{I}_{n}^{*}=\left\{k_{n}^{*}+1, \ldots, m_{n}^{*}\right\}$. The value $a_{n}$ during the period $\mathbb{I}_{n}^{*}$ is interpreted as an epidemic deviation from the usual (zero) mean of innovations, and $\ell_{n}^{*}=$ $m_{n}^{*}-k_{n}^{*}$ is the duration of the epidemic state. Note that Markevičiūte et al. [15] investigated uniform increments statistics built on observations for the nearly nonstationary process. Since innovations are not observed, we may build a uniform increments statistics also on least squares residuals. Set for $\alpha \in[0,1)$

$$
\widehat{T}_{\alpha, n}=T_{\alpha, n}\left(\widehat{\varepsilon}_{1}, \ldots, \widehat{\varepsilon}_{n}\right)=\max _{1 \leqslant \ell \leqslant n} \ell^{-\alpha} \max _{1 \leqslant k \leqslant n-\ell}\left|\sum_{j=k+1}^{k+\ell} \widehat{\varepsilon}_{j}-\frac{\ell}{n} \sum_{j=1}^{n} \widehat{\varepsilon}_{j}\right|,
$$

where $\left(\widehat{\varepsilon}_{k}\right)$ are residuals of the model (5.]) defined by ([L.3) and (‥4).

Using the Hölderian limit theorems, it is easy to find the limit of test statistics (5.2) under the null hypothesis. Note that the test statistics for the second type model with different approach is investigated in detail in Markevičiūte et al. [16], so we find the limit under the null hypothesis only for the first type model, and we assume that all $a_{n, k}=0$.

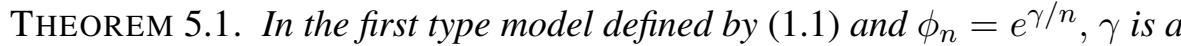
negative constant. Assume that innovations satisfy $\lim _{t \rightarrow \infty} t^{p} \mathbb{P}\left(\left|\varepsilon_{0}\right|>t\right)=0$ for some $p>2$. Then under $H_{0}$ for any $\alpha \in(0,1 / 2-1 / p)$

$$
n^{-1 / 2+\alpha} \sigma^{-1} \widehat{T}_{\alpha, n} \underset{n \rightarrow \infty}{\stackrel{\mathcal{D}}{\longrightarrow}} T_{\alpha, \infty}(Z),
$$

where $\sigma^{2}=\mathbb{E} \varepsilon_{1}^{2}$, and

$$
Z(t)=W(t)-A^{-1} B J(t)
$$

Pro o f. Using Lemma A.1 in [15] and Theorem [.], we immediately obtain the result.

5.1.1. Test power analysis. In this section we perform the test power analysis.

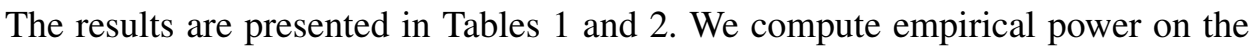
size-adjusted (not nominal size) basis, i.e., we replace the nominal value of significance level by the value of empirical distribution function for $p$-values under the null hypothesis.

Here we compute $N=1000$ realizations of test statistics with the sample size $n$ for different values of parameters $\gamma, \gamma_{n}, \alpha, k^{*}, \ell^{*}$ and $a_{n}$. Innovations $\left(\varepsilon_{j}\right)$ are generated as standard normally distributed random variables. For the limit distribution we compute $N=5000$ realizations of test statistics with the sample size $n=5000$. We approximate the values of the standard Wiener process by

$$
W\left(\frac{k}{5000}\right)=5000^{-1 / 2} \sum_{j=1}^{k} \varepsilon(j), \quad k=1, \ldots, 5000 .
$$


TABLE 1. Empirical power at the size-adjusted significance level 0.05 for the first type model with Gaussian innovations

\begin{tabular}{|l|ccc|}
\hline Parameters & $\alpha=2 / 32$ & $\alpha=6 / 32$ & $\alpha=12.5 / 32$ \\
\hline$\ell^{*} / n=0.035$ & 0.462 & 0.715 & 0.968 \\
$\ell^{*} / n=0.050$ & 0.879 & 0.981 & 0.998 \\
$\ell^{*} / n=0.065$ & 0.988 & 1.000 & 1.000 \\
\hline$k^{*} / n=0.2$ & 0.903 & 0.981 & 1.000 \\
$k^{*} / n=0.4$ & 0.879 & 0.981 & 0.998 \\
$k^{*} / n=0.8$ & 0.784 & 0.967 & 0.997 \\
\hline$a_{n}=0.8$ & 0.574 & 0.793 & 0.957 \\
$a_{n}=1$ & 0.879 & 0.981 & 0.998 \\
$a_{n}=1.2$ & 0.989 & 1.000 & 1.000 \\
\hline$n=500$ & 0.498 & 0.700 & 0.884 \\
$n=1000$ & 0.879 & 0.981 & 0.998 \\
$n=2000$ & 1.000 & 1.000 & 1.000 \\
\hline$\gamma=-2$ & 0.879 & 0.981 & 0.998 \\
$\gamma=-12$ & 0.831 & 0.976 & 0.998 \\
$\gamma=-100$ & 0.010 & 0.267 & 0.975 \\
\hline
\end{tabular}

TABLE 2. Empirical power at the size-adjusted significance level 0.05 for the second type model with Gaussian innovations

\begin{tabular}{|l|ccc|}
\hline Parameters & $\alpha=2 / 32$ & $\alpha=6 / 32$ & $\alpha=10 / 32$ \\
\hline$\ell^{*} / n=0.035$ & 0.049 & 0.190 & 0.763 \\
$\ell^{*} / n=0.050$ & 0.093 & 0.573 & 0.965 \\
$\ell^{*} / n=0.065$ & 0.216 & 0.880 & 0.998 \\
\hline$k^{*} / n=0.2$ & 0.077 & 0.589 & 0.974 \\
$k^{*} / n=0.4$ & 0.093 & 0.573 & 0.965 \\
$k^{*} / n=0.8$ & 0.105 & 0.615 & 0.974 \\
\hline$a_{n}=0.8$ & 0.102 & 0.328 & 0.791 \\
$a_{n}=1$ & 0.093 & 0.573 & 0.965 \\
$a_{n}=1.2$ & 0.080 & 0.810 & 1.000 \\
\hline$n=500$ & 0.062 & 0.171 & 0.552 \\
$n=1000$ & 0.093 & 0.573 & 0.965 \\
$n=2000$ & 0.660 & 0.997 & 1.000 \\
\hline$\gamma_{n}=n / \ln (n)$ & 0.035 & 0.416 & 0.950 \\
$\gamma_{n}=\ln ^{2.5}(n)$ & 0.020 & 0.353 & 0.935 \\
$\gamma_{n}=n^{3 / 4}$ & 0.093 & 0.573 & 0.965 \\
\hline
\end{tabular}

The Ornstein-Uhlenbeck process has been approximated by the following discretization:

$$
S(j)=S(j-1) e^{\gamma / n}+\sqrt{\frac{1-e^{2 \gamma / n}}{-2 \gamma}} \cdot \varepsilon(j), \quad \varepsilon(j) \sim \mathfrak{N}(0,1) .
$$


Using values generated by (5.5), we approximate the integrated Ornstein-Uhlenbeck process by

$$
J\left(\frac{k}{5000}\right)=5000^{-1} \sum_{j=1}^{k} S(j), \quad k=1, \ldots, 5000,
$$

and values

$$
A=5000^{-1} \sum_{j=1}^{n} S^{2}(j), \quad B=\sum_{j=1}^{n} S(j)\left(W\left(\frac{j}{5000}\right)-W\left(\frac{j-1}{5000}\right)\right) .
$$

For the first type model $\left(\phi_{n}=e^{\gamma / n}\right)$ with innovations that satisfy the integrability condition $(2.3])$ the basic parameters are

$$
\gamma=-2, \quad a_{n}=1, \quad n=1000, \quad \frac{\ell^{*}}{n}=0.05, \quad \frac{k^{*}}{n}=0.4, \quad y_{n, 0}=0 .
$$

We modify them separately and compute the empirical size-power. We keep all the parameters fixed except one (indicated in the first column in both tables), which is allowed to vary.

As one can see in Table $\square$ the test power increases with the $\alpha$. The test statistics has a quite big power in detecting short epidemics with $\alpha$ closer to $1 / 2$. Naturally, increasing $n$ increases the test power. In general, the test has a quite big power for all chosen parameters.

For the second type model $\left(\phi_{n}=1-\gamma_{n} / n\right)$ with innovations that satisfy the integrability condition (2.3), the basic parameters are

$$
\gamma_{n}=n^{3 / 4}, \quad a_{n}=1, \quad n=1000, \quad \frac{\ell^{*}}{n}=0.05, \quad \frac{k^{*}}{n}=0.4, \quad y_{n, 0}=0 .
$$

For the second type model (Table $\mathbb{Z}$ ), the test power is very low for the small $\alpha$. The test power increases with $n, \ell^{*}$ and the rate of divergence of $\gamma_{n}$.

5.2. Comparison with other test statistics. Table B shows size-adjusted test power for statistics $\widetilde{T}_{\alpha, n}=T_{\alpha, n}\left(y_{n, 1}, \ldots, y_{n, n}\right)$ (see [15]) and statistics $\widehat{T}_{\alpha, n}=$ $T_{\alpha, n}\left(\widehat{\varepsilon}_{1}, \ldots, \widehat{\varepsilon}_{n}\right)$ (see also [16]), where innovations satisfy the integrability condition ([2.3). The result shows that with different parameters for the second type model, both statistics give opposite results. In this example, statistics $\widehat{T}_{\alpha, n}$ with $\gamma_{n}=n^{0.45}$ detects epidemics better, while statistics $\widetilde{T}_{\alpha, n}$ performs better with $\gamma_{n}=n^{0.8}$

Acknowledgments. The author would like to express her special gratitude to Charles Suquet for useful discussions. Also, the author would like to thank the referee for the very careful reading and valuable remarks. 
TABLE 3. Comparison of the statistics $\widetilde{T}_{\alpha, n}$ and $\widehat{T}_{\alpha, n}$ for the first type model (model I) and for the second type model (model II)

\begin{tabular}{|c|c|c|c|c|}
\hline \multicolumn{5}{|c|}{$a_{n}=1, \ell^{*}=30, k^{*}=400, n=1000, \gamma=-2, \gamma_{n}=n^{0.45}$} \\
\hline \multicolumn{2}{|c|}{} & $\alpha_{1}=0.0625$ & $\alpha_{2}=0.1875$ & $\begin{array}{c}\alpha_{3}=0.39 \text { (model I) } \\
\alpha_{3}=0.31 \text { (model II) }\end{array}$ \\
\hline \multirow{2}{*}{$\widetilde{T}_{\alpha, n}$} & model I & 0.318 & 0.327 & 0.306 \\
& model II & 0.276 & 0.330 & 0.429 \\
\hline \multirow{2}{*}{$\widehat{T}_{\alpha, n}$} & model I & 0.335 & 0.526 & 0.914 \\
& model II & 0.061 & 0.452 & 0.836 \\
\hline \multicolumn{2}{|c|}{$a_{n}=1, \ell^{*}=30, k^{*}=400, n=1000, \gamma=-20, \gamma_{n}=n^{0.8}$} \\
\hline$\widetilde{T}_{\alpha, n}$ & model I & 0.280 & 0.322 & 0.467 \\
& model II & 0.314 & 0.505 & 0.796 \\
\hline \multirow{T}{*}{$\widehat{T}_{\alpha, n}$} & model I & 0.088 & 0.502 & 0.913 \\
& model II & 0.073 & 0.213 & 0.682 \\
\hline
\end{tabular}

\section{REFERENCES}

[1] P. J. Avery and A. D. Henderson, Detecting a changed segment in DNA sequences, J. R. Stat. Soc. Ser. C. Appl. Stat. 48 (1999), pp. 489-503.

[2] J. B ai, On the partial sums of residuals in autoregressive and moving average models, J. Time Series Anal. 14 (1993), pp. 247-260.

[3] P. Billingsley, Convergence of Probability Measures, Wiley, New York 1968.

[4] L. Broemeling and H. Tsurumi, Econometrics and Structural Change, Marcel Dekker, New York 1987.

[5] D. Commenges, J. Seal, and F. Pinatel, Inference about a change point in experimental neurophysiology, Math. Biosci. 80 (1986), pp. 81-108.

[6] L. Giraitis and P. C. B. Phillips, Uniform limit theory for stationary autoregression, J. Time Series Anal. 27 (1) (2006), pp. 51-60.

[7] E Gombay, Testing for change-points with rank and sign statistics, Statist. Probab. Lett. 20 (1994), pp. 49-55.

[8] L. Horváth, Change in autoregressive process, Stochastic Process. Appl. 44 (1993), pp. 221 242.

[9] V. K. Jandhyala and I. B. MacNeil, Iterated partial sum sequences of regression residuals and tests for change points with continuity constraints, J. R. Stat. Soc. Ser. B 59 (1997), pp. 147-156.

[10] M. Juodis, A. Račkauskas, and Ch. Suquet, Hölderian functional central limit theorems for linear processes, ALEA Lat. Am. J. Probab. Math. Stat. 5 (2009), pp. 47-64.

[11] R. J. Kulperger, On the residuals of autoregressive processes and polynomial regression, Stochastic Process. Appl. 21 (1) (1985), pp. 107-118.

[12] R. J. Kulperger and H. Yu, High moment partial sum processes of residuals in GARCH models and their applications, Ann. Statist. 33 (2005), pp. 2395-2422.

[13] B. Levin and J. Kline, The CUSUM test of homogeneity with an application in spontaneous abortion epidemiology, Stat. Med. 4 (1985), pp. 469-488.

[14] J. Markevičiūtė, A. Račkauskas, and Ch. Suquet, Functional limit theorems for sums of nearly nonstationary processes, Lith. Math. J. 52 (3) (2012), pp. 282-296.

[15] J. Markevičiūtè, A. Račkauskas, and Ch. Suquet, Testing the epidemic change in nearly nonstationary autoregressive processes, Nonlinear Anal. Model. Control 19 (1) (2014), pp. 67-82. 
[16] J. Markevičiūtè, A. Račkauskas, and Ch. Suquet, Testing epidemic change in nearly nonstationary process with statistics based on residuals, Statist. Papers, doi: 10.1007/s00362015-0712-0, 2015.

[17] N. Mimoto, Convergence in distribution for the sup-norm of a kernel density estimator for GARCH innovations, Statist. Probab. Lett. 78 (2008), pp. 915-923.

[18] P. C. B. Phillips, Towards a unified asymptotic theory for autoregression, Biometrika 74 (3) (1987), pp. 535-547.

[19] A. Račkauskas and I Rastenè, Hölder convergence of autoregression residuals partial sum processes, Lith. Math. J. 48 (4) (2008), pp. 438-450.

[20] A. Račkauskas and Ch. Suquet, Necessary and sufficient condition for the functional central limit theorem in Hölder spaces, J. Theoret. Probab. 17 (1) (2004), pp. 221-243.

[21] A. Račkauskas and Ch. Suquet, Hölder norm test statistics for epidemic change, J. Statist. Plann. Inference 126 (2) (2004), pp. 495-520.

[22] X. Shen, Z. Lin, and C. Zhang, Asymptotic distributions of innovation density estimators in linear processes, Comm. Statist. Theory Methods 37 (2008), pp. 2262-2275.

[23] D. W. Shin, The limiting distribution of the residual processes in nonstationary autoregressive processes, J. Time Series Anal. 19 (6) (1998), pp. 723-736.

[24] Ch. Suquet, Tightness in Schauder decomposable Banach spaces, Amer. Math. Soc. Transl. (2) 193 (1999), pp. 201-224.

[25] H. Yu, High moment partial sum processes of residuals in ARMA models and their applications, J. Time Series Anal. 28 (2007), pp. 72-91.

Jurgita Markevičiūtė

Faculty of Mathematics and Informatics

Vilnius University

Naugarduko str. 24

LT-03225 Vilnius, Lithuania

E-mail: jurgita.markeviciute@mif.vu.lt

Received on 5.12.2014;

revised version on 4.8 .2016 